\title{
Impact of COVID-19 shelter-in-place order on transmission of gastrointestinal pathogens in Northern California
}

Philip L. Bulterys ${ }^{1}$, Nicole Y. Leung² ${ }^{2}$ Atif Saleem ${ }^{1}$, Indre Budvytiene ${ }^{4}$, Niaz Banaei ${ }^{1,3,4}$

${ }^{1}$ Department of Pathology, Stanford University School of Medicine, Stanford, CA, USA

${ }^{2}$ Department of Psychiatry and Behavioral Sciences, Stanford University School of Medicine, Stanford, CA, USA

${ }^{3}$ Department of Medicine, Division of Infectious Diseases and Geographic Medicine, Stanford University, Stanford, CA, USA

${ }^{4}$ Clinical Microbiology Laboratory, Stanford University Medical Center, Stanford, CA, USA

To whom correspondence should be addressed:

Niaz Banaei, M.D.

3375 Hillview Ave, Rm. 1602

Palo Alto, CA 94304

Phone 650-725-8052

Fax 650-725-5671

nbanaei@stanford.edu 


\section{Abstract}

Society-wide cessation of human interaction outside the household due to the COVID-19 shelter-in-place created a unique opportunity in modern history to reexamine the transmission of communicable gastrointestinal pathogens. We conducted a quasi-experimental study from January 1, 2018 to Sept 30, 2020 to investigate the effect of California's COVID-19 shelter-inplace order on the community transmission of viral, bacterial, and parasitic gastrointestinal pathogens detected with the FilmArray GI Panel (BioFire Diagnostics, Salt Lake City, UT). The incidence of viral causes of gastroenteritis, enteroaggregative/enteropathogenic/enterotoxigenic Escherichia coli, Shigella, and Cyclospora cayetanensis decreased sharply after shelter-in place took effect, whilst Salmonella, Campylobacter, shiga toxin-producing E. coli (O157 and nonO157) and other bacterial and parasitic causes of gastroenteritis were largely unaffected. Findings suggest community spread of viral gastroenteritis, pathogenic $E$. coli (except for shiga toxin-producing E. coli), Shigella, and Cyclospora is more susceptible to changes associated with shelter-in-place than other gastrointestinal pathogens.

Keywords: Gastrointestinal pathogen; gastroenteritis; COVID-19; shelter-in-place; community transmission 


\section{Brief Report}

In response to the COVID-19 pandemic, California was the first state to impose a strict shelterin-place (SIP) order in March 2020 (1). Although enforced social distancing early in the pandemic appears to have delayed the spread of COVID-19 $(1,2)$, little is known about its potential impact on the incidence of other communicable infectious diseases. Such a natural experiment involving the society-wide cessation of human interaction outside the household is unique in modern history and could provide useful insight regarding transmission patterns of other pathogens circulating in the community. The objective of this study was to determine the impact of California's SIP order on the gastrointestinal pathogen landscape dynamics in Northern California.

The FilmArray GI Panel (BioFire Diagnostics, Salt Lake City, UT) is a multiplex, on-demand, sample-to-answer, real-time PCR assay for the syndromic diagnosis of infectious gastroenteritis (3). We analyzed all FilmArray GI Panel results for adult and pediatric patients at the Stanford Health Care Clinical Microbiology laboratory between January 1, 2018 and September 30, 2020. For inpatients, rejection criteria included excluding patients that had been hospitalized for more than 72 hours. We calculated odds ratios and performed Fisher's exact tests to compare the test positivity rates of all 22 pathogens represented on the panel before and after March 20, 2020, when California's SIP order was instituted. The study was approved by the Stanford Institutional Review Board.

In total, 10,317 tests were performed during the study period (from 8,321 unique patients) and were included in the analysis. Of these, 8,677 were performed before SIP and 1,640 were performed after SIP. The average number of tests performed per quarter for the entire study period (January 1, 2018-September 30, 2020) was 938 (see Figure 1). Of all tests performed before SIP ( $\mathrm{n}=8,677), 4,792(55 \%)$ were from patients admitted to a Stanford-affiliated 
hospital, and $3,885(45 \%)$ were from patients seen in the outpatient setting. Of all tests performed after SIP ( $n=1,640), 1,045(64 \%)$ were from inpatients, and $595(36 \%)$ from outpatients.

When comparing rates of infection before SIP (January 1, 2018- March 19, 2020) to after SIP (March 20, 2020- September 30, 2020), we found that the incidence of viral causes of gastroenteritis, including adenovirus F40/41, astrovirus, norovirus, rotavirus, and sapovirus, was markedly decreased after shelter-in place took effect (see Figure 1). Adenovirus F40/41 detection decreased by a factor of $13(p=0.0004)$, astrovirus by a factor of eight $(p<0.0001)$, norovirus by a factor of five $(p<0.0001)$, rotavirus by a factor of two $(p=0.03)$, and sapovirus by a factor of eight $(p<0.0001)$ (see Table 1). All pathogenic Escherichia coli types (with the exception of shiga toxin-producing E. coli), Shigella species, and Cyclospora cayetanensis were also detected significantly less frequently after shelter-in-place. The incidence of enteroaggregative $E$. coli decreased by a factor of four $(p<0.0001)$, enteropathogenic $E$. coli by a factor of two $(p<0.0001)$, enterotoxigenic $E$. coli by a factor of three $(p<0.0001)$, Shigella species by a factor of two $(p=0.033)$, and Cyclospora cayetanensis positivity rates decreased from $0.51 \%$ to $0 \%(p=0.0019)$. The incidence of Plesiomonas shigelloides, Cryptosporidium species, Entamoeba histolytica, and Giardia lamblia also decreased, but the changes were not statistically significant. The incidence of other gastrointestinal pathogens, including Campylobacter species, Clostridium difficile, Salmonella enterica, shiga toxin-producing E. coli (O157 and non-O157), Vibrio cholerae and other Vibrio species, and Yersinia enterocolitica, was largely unaffected.

To our knowledge, this is the first study to comprehensively evaluate the impact of COVID-19 pandemic-related social distancing measures on the gastrointestinal pathogen landscape. SIP orders and practice resulted in a notable decrease in human-to-human contact outside of 
households, with the closure of most workplaces, schools, and day cares, confinement of much of the population to their homes, as well as reduced travel (4). Although little data on food consumption patterns during SIP exist, we presume that the consumption of store-bought (including online purchases) and prepared restaurant foods (take-out and outdoor dining) largely persisted (5). In the absence of data to indicate significant changes in diet or food source, the altered gastrointestinal pathogen landscape that we observe is likely largely a result of decreased human-human contact and reduced travel. Although all the pathogens on the Biofire GI PCR panel are transmitted by the fecal-oral route, our findings suggest previously unappreciated differences in the transmission of these pathogens. Specifically, our data suggest that community spread of viral gastroenteritis, pathogenic E. coli (except for shiga toxinproducing E. coli), Shigella, and Cyclospora are more susceptible to changes associated with shelter-in-place than other gastrointestinal pathogens.

For adenovirus F40/41, astrovirus, norovirus, rotavirus, and sapovirus, transmission via personto-person contact is well established (6). These pathogens often afflict daycares, nurseries, military barracks, and long-term care facilities, where personal contact is inevitable (6). It is conceivable that the closure of daycares and nurseries significantly reduced community transmission of these viruses, both among children and their close household contacts. Pathogenic E. coli and Shigella, on the other hand, are thought to be transmitted primarily through contaminated foods (7). Most outbreaks have been traced to specific foods, such as store-bought lettuce, flour, or ground beef (7). Our finding that these species (except for shiga toxin-producing E. coli) were detected significantly less frequently following SIP may reflect reduced travel-associated consumption of contaminated foods. In contrast, shiga toxinproducing strains of E. coli (O157 and non-O157), which are present in US-manufactured foods (8), were unaffected $(7,9)$. Similarly, incidence of the prototypical food-borne intestinal 
pathogens, Campylobacter and Salmonella, was unaffected, suggesting continuous production and consumption of contaminated foods.

We observed decreases in the rates of Plesiomonas shigelloides, Cryptosporidium species, Entamoeba histolytica, and Giardia lamblia rates following SIP. These differences were not statistically significant, and we attribute this to the relatively low frequency of these pathogens pre-SIP (see Figure 1). The decreased rates of these pathogens likely reflect reduced humanhuman contact, outdoor recreation, and travel, as contact with infected persons, exposure to contaminated recreational or drinking water, and travel to highly endemic areas are known risk factors for these pathogens (10-12). We observed a slight (12\%) increase in the incidence of Clostridium difficile following SIP, which was not statistically significant $(p=0.23)$. Studies assessing the effect of COVID-19 on the incidence of $C$. difficile have focused on the hospital setting and reached differing conclusions. Ponce-Alonso et al (13) found that the rates of nosocomial $C$. difficile decreased by $70 \%$ in a tertiary care hospital in Spain during the COVID19 pandemic, while Lewandowski et al (14) observed a four-fold increase in C. difficile infection at a hospital in Poland during the pandemic.

Cyclospora cayetanensis, the etiologic agent of cyclosporiasis, is a parasite thought to be transmitted via contaminated food or water (15). Infected individuals shed unsporulated (noninfective) oocysts in their stool, which require 1-2 weeks in favorable environmental conditions to sporulate and become infective (15). It is therefore thought that person-to-person transmission is rare. Most outbreaks in the United States have been linked to imported fresh produce, such as raspberries, basil, and snow peas (16). Thus, the decrease in the incidence of Cyclospora cayetanensis following SIP is most likely attributable to reduced travel, although reduced consumption of imported fresh produce as a result of disrupted food supply chains may 
have also contributed (17). Further epidemiological investigation will be required to test this hypothesis.

In summary, COVID-19 SIP measures represent a unique opportunity to reexamine our understanding of the transmission of infectious agents causing gastroenteritis. We identified shifts in the incidence of gastrointestinal pathogens following COVID-19 SIP that may shed new light on the transmission of these pathogens and provide opportunities to test novel strategies to disrupt transmissions of these pathogens. This study is limited in that it did not include patients with gastroenteritis that did not seek medical care and thus did not receive a FilmArray GI panel. The findings may be different if all patients with gastroenteritis were included. In addition, only cases dating back to January 2018 were included, precluding an in-depth analysis of annual season trends. The study is strengthened by a large sample size (>10,000 GI PCR assays), the unique circumstance of the California SIP, and the ability to precisely pinpoint the timing of SIP intervention. Additional studies are needed to better characterize the effect of COVID-19 and mitigation strategies on the transmission of gastrointestinal pathogens. 


\section{References}

1. Lyu W, Wehby GL. Shelter-In-Place Orders Reduced COVID-19 Mortality And Reduced The Rate Of Growth In Hospitalizations. Health Aff (Millwood). 2020;39(9):1615-23.

2. Islam N, Sharp SJ, Chowell G, Shabnam S, Kawachi I, Lacey B, et al. Physical distancing interventions and incidence of coronavirus disease 2019: natural experiment in 149 countries. BMJ. 2020;370:m2743.

3. Murphy CN, Fowler RC, Iwen PC, Fey PD. Evaluation of the BioFire FilmArray(R) GastrointestinalPanel in a Midwestern Academic Hospital. Eur J Clin Microbiol Infect Dis. 2017;36(4):747-54.

4. Jacobsen GD JK. Statewide COVID-19 Stay-at-Home Orders and Population Mobility in the United States. World Medical and Health Policy. 2020.

5. Eftimov T, Popovski G, Petkovic M, Seljak BK, Kocev D. COVID-19 pandemic changes the food consumption patterns. Trends Food Sci Technol. 2020;104:268-72.

6. Orenstein R. Gastroenteritis, Viral. Encyclopedia of Gastroenterology. 2019:652-7.

7. Croxen MA, Law RJ, Scholz R, Keeney KM, Wlodarska M, Finlay BB. Recent advances in understanding enteric pathogenic Escherichia coli. Clin Microbiol Rev. 2013;26(4):822-80.

8. White A, Cronquist A, Bedrick EJ, Scallan E. Food Source Prediction of Shiga ToxinProducing Escherichia coli Outbreaks Using Demographic and Outbreak Characteristics, United States, 1998-2014. Foodborne Pathog Dis. 2016;13(10):527-34.

9. Bryan A, Youngster I, McAdam AJ. Shiga Toxin Producing Escherichia coli. Clin Lab Med. 2015;35(2):247-72.

10. Einarsson E, Ma'ayeh S, Svard SG. An up-date on Giardia and giardiasis. Curr Opin Microbiol. 2016;34:47-52.

11. Janda JM, Abbott SL, Mclver CJ. Plesiomonas shigelloides Revisited. Clin Microbiol Rev. 2016;29(2):349-74.

12. Gharpure R, Perez A, Miller AD, Wikswo ME, Silver R, Hlavsa MC. Cryptosporidiosis Outbreaks - United States, 2009-2017. MMWR Morb Mortal Wkly Rep. 2019;68(25):56872.

13. Ponce-Alonso M, Saez de la Fuente J, Rincon-Carlavilla A, Moreno-Nunez P, MartinezGarcia L, Escudero-Sanchez R, et al. Impact of the coronavirus disease 2019 (COVID19) pandemic on nosocomial Clostridioides difficile infection. Infect Control Hosp Epidemiol. 2020:1-5. 
14. Lewandowski K, Rosolowski M, Kaniewska M, Kucha P, Meler A, Wierzba W, et al. Clostridioides difficile infection in coronavirus disease 2019: an underestimated problem? Pol Arch Intern Med. 2020.

15. Ortega YR, Sanchez R. Update on Cyclospora cayetanensis, a food-borne and waterborne parasite. Clin Microbiol Rev. 2010;23(1):218-34.

16. Li J, Wang R, Chen Y, Xiao L, Zhang L. Cyclospora cayetanensis infection in humans: biological characteristics, clinical features, epidemiology, detection method and treatment. Parasitology. 2020;147(2):160-70.

17. Aday S AM. Impacts of COVID-19 on food supply chain. Food Quality and Safety. 2020. 
Figure 1. GI PCR positivity rates before and after implementation of California's shelterin-place, March 20, 2020. Shown are the quarterly positivity rate (positives/tests) for all gastrointestinal pathogens represented on the FilmArray GI panel from January 1, 2018 to September 30, 2020. The blue line in each plot indicates test positivity rate, whereas the grey line indicates test volume (total tests per quarter). The dotted red line designates when California's shelter-in-place was instituted (March 20, 2020). The plots are organized with viruses appearing first, then bacteria, then parasites. 
medRxiv preprint doi: https://doi.org/10.1101/2021.01.12.21249708; this version posted January 15, 2021. The copyright holder for this preprint (which was not certified by peer review) is the author/funder, who has granted medRxiv a license to display the preprint in perpetuity. It is made available under a CC-BY-NC-ND 4.0 International license.

$\stackrel{\text { の }}{\stackrel{\text { ભ }}{>}}$
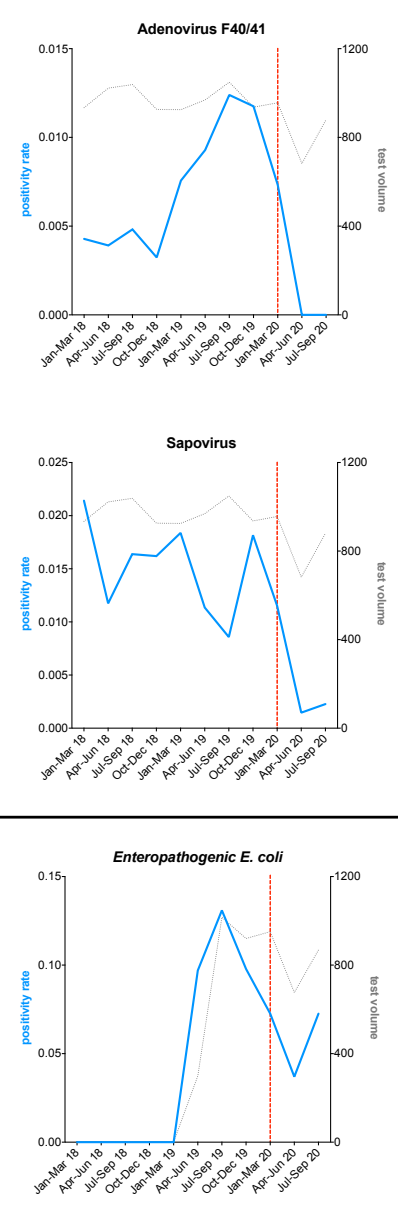

造
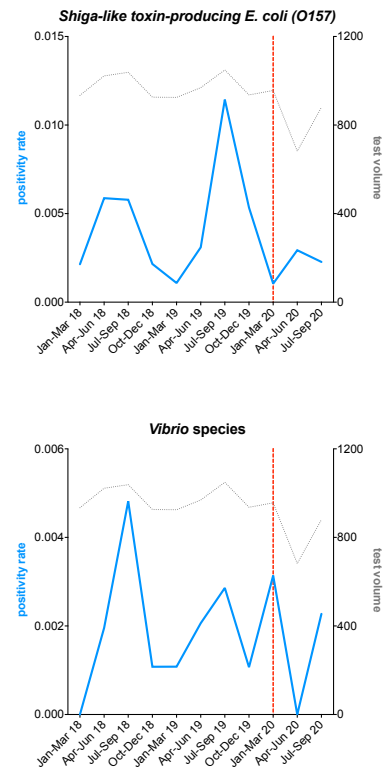

㟶

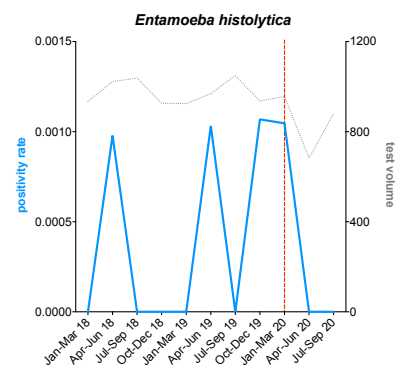

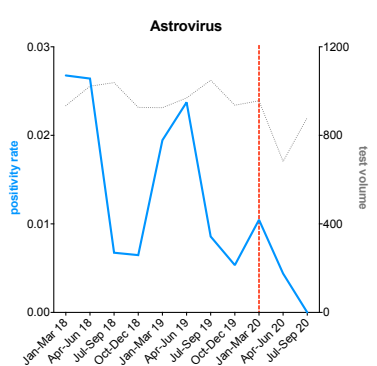
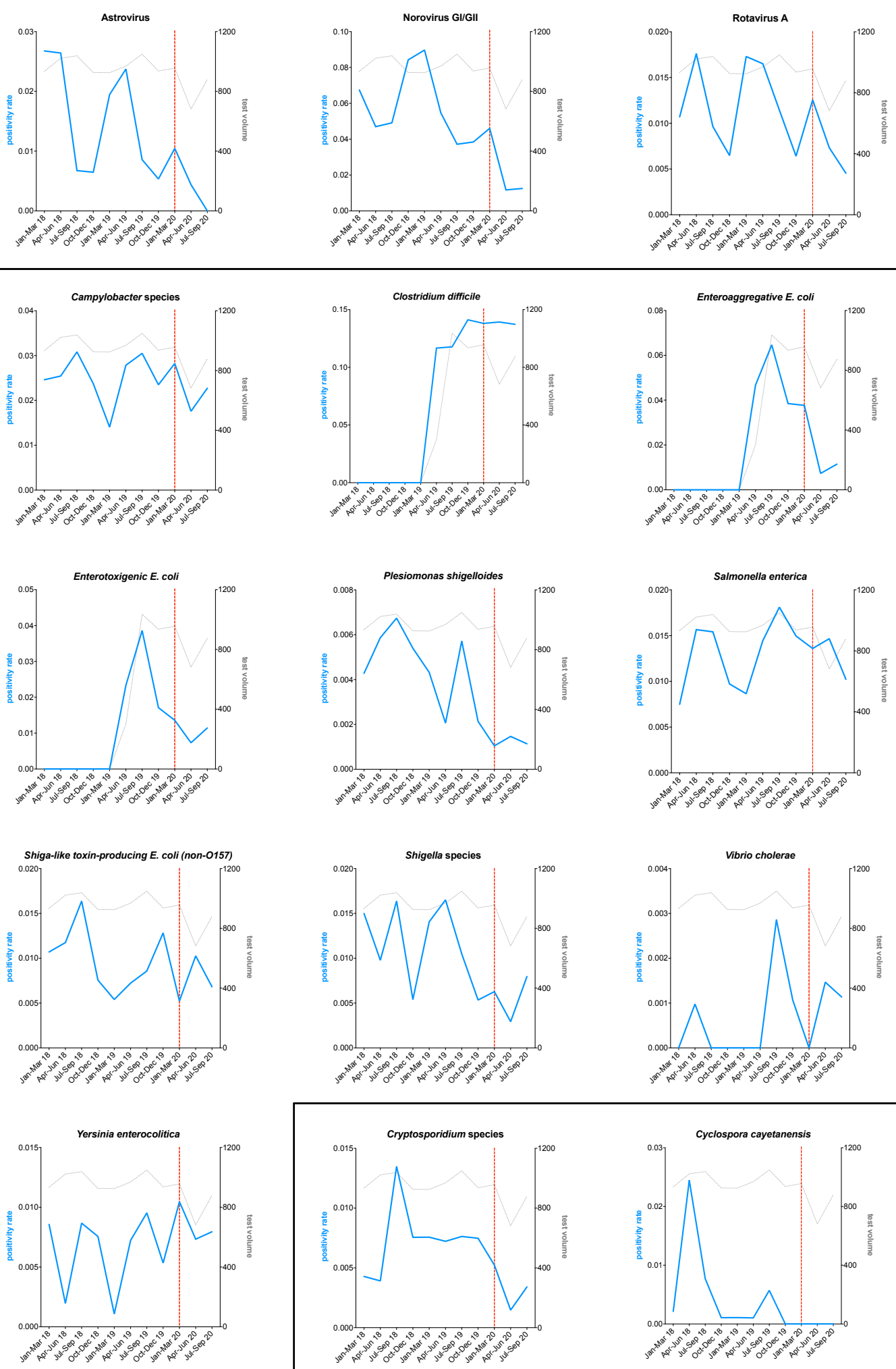
medRxiv preprint doi: https://doi.org/10.1101/2021.01.12.21249708; this version posted January 15, 2021. The copyright holder for this preprint

(which was not certified by peer review) is the author/funder, who has granted medRxiv a license to display the preprint in perpetuity.

It is made available under a CC-BY-NC-ND 4.0 International license.

Table 1. The odds of detection of gastrointestinal pathogens before and after implementation of California's shelter-in-place, March 20, 2020. Shown for each pathogen

on the FilmArray GI panel are the total number of tests performed, the test positivity rates before and after shelter-in-place was instituted, the odds ratio and $95 \%$ confidence interval $(\mathrm{Cl})$ comparing the odds of test positivity before SIP/after SIP, and P value calculated using a Fisher's exact test. Odds ratios were calculated using the Baptista Pike method. All statistical comparisons were implemented in GraphPad Prism version 9.0.0. 


\section{$\underline{\text { Total tests }}$}

Before SIP After SIP

positives/tests (\%)

\section{VIRUS}

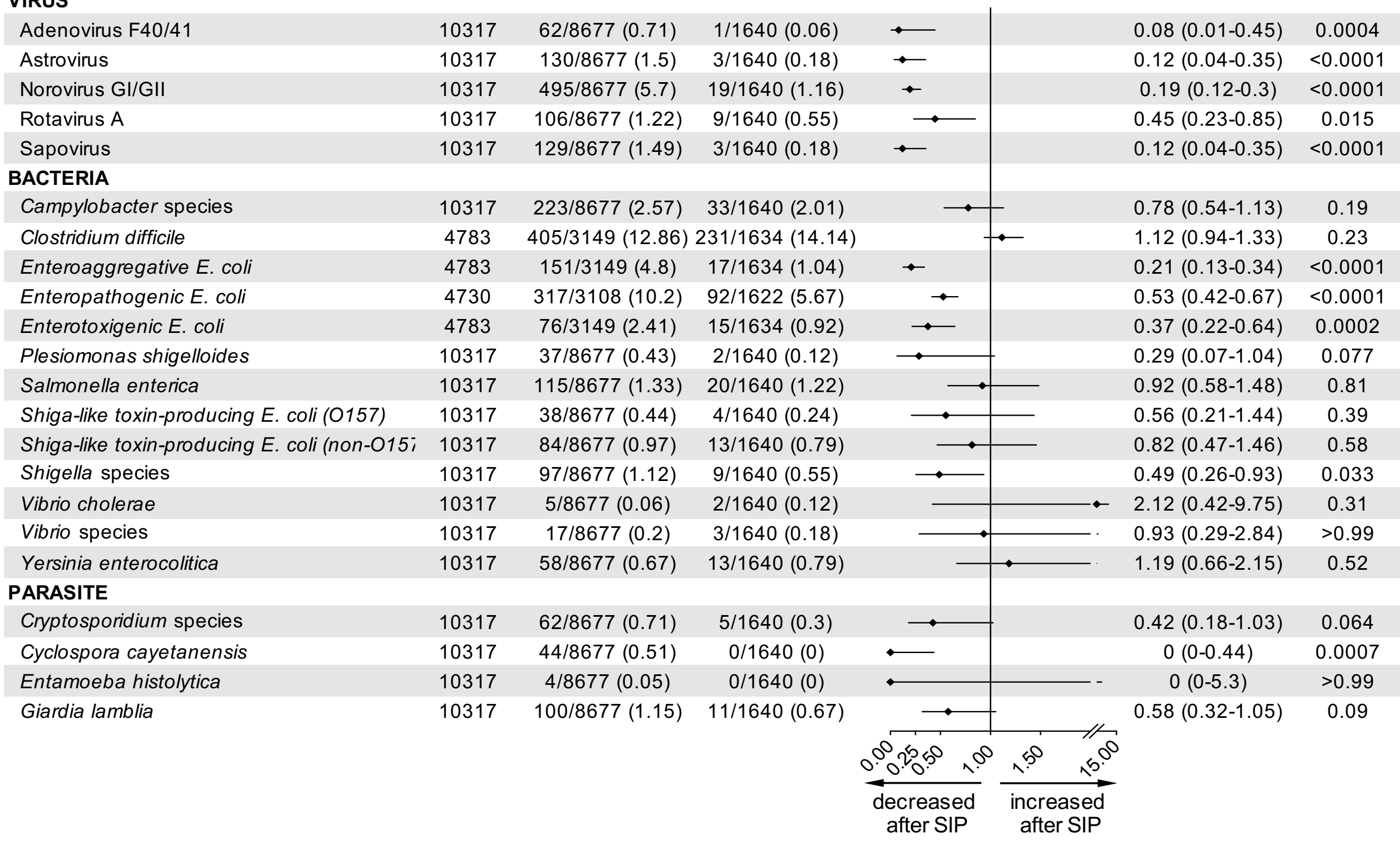

